\title{
Density-matrix approach to wave-packet dynamics
}

\author{
M. Rosina \\ Faculty of Natural Sciences and Technology, University of Ljubljana and J. Stefan Institute, Ljubljana, Yugoslavia \\ P. Van Leuven \\ Dienst Theoretische en Wiskundige Natuurkunde, University of Antwerp, Groenenborgerlaan 171, B2020 Antwerpen, Belgium
}

(Received 15 July 1991)

\begin{abstract}
We investigate the dynamics of the second-order moments of wave packets by means of density-matrix techniques. In particular, we consider the existence of shape-conserving pure states and ensembles.
\end{abstract}

PACS number(s): 03.65.Ca, 03.65.Sq

\section{INTRODUCTION}

In nonrelativistic quantum mechanics the time propagation of coherent-state wave packets representing a single particle was first used to demonstrate their semiclassical properties. Since the work of Schrödinger [1] there have been at least two revivals of the subject: one with the Glauber coherent states in the context of quantum optics [2], and, recently, one with the definition of generalized coherent states in the context of group theory [3]. In atomic and molecular physics, the latter revival is connected with the development of experiments in the picosecond and femtosecond range, whereas in other fields, such as nuclear physics, it is related to conceptual aspects of the collective behavior of the many-particle systems.

Recently [4] attention has been drawn to the fact that the nonspreading property of the Glauber state can be generalized to other forms of shape conservation. Families of Gaussian wave packets can be defined, which, during propagation in a harmonic-oscillator potential, conserve their ellipsoidal shape and move as a uniformly rotating rigid probability distribution.

In this paper we study the subject of wave-packet propagation, in particular the shape conservation, from a more general point of view. Although for the sake of illustration we shall still consider a single-particle state, we wish to extend the discussion to non-Gaussian-type wave functions. We use the density-matrix formalism because it provides an elegant and powerful theoretical structure adapted to the analysis of the wave function in terms of moments. In this formalism it is then straightforward to take also mixed states or ensembles into consideration.

Let us call the "reduced density matrix" the matrix

$$
D_{a b}=\operatorname{Tr}\left(\rho A_{a}^{\dagger} A_{b}\right)
$$

or

$$
D_{a b}=\left\langle\psi\left|A_{a}^{\dagger} A_{b}\right| \psi\right\rangle,
$$

where $A_{a}$ is an arbitrary set of operators and $\rho$ is the density operator in the case of an ensemble or $|\psi\rangle$ is the wave function in the case of a pure state. With a finite set of operators $\boldsymbol{A}_{a}$, the density matrix contains of course only partial information about the wave function or en- semble.

We shall be interested in a set of operators $A_{a}$ which can be expressed as polynomials of the position operators $x_{i}$ and momentum operators $p_{i}, i=x, y, z$. Throughout the paper we shall use dimensionless quantities, i.e., $x_{i}$ will be expressed in units of some basic length parameter $b$ and $p_{i}$ in units $\hbar / b$. We choose our set of operators $A_{a}=\left\{1, x_{i}, p_{i}\right\}$ and write the reduced density matrix

$$
\left(\begin{array}{ccc}
1 & \bar{x} & \bar{p} \\
\bar{x} & S & Q \\
\bar{p} & Q^{\dagger} & K
\end{array}\right) .
$$

Here we have defined the matrix elements as follows:

$$
\begin{aligned}
& \bar{x}_{i}=\left\langle\psi\left|x_{i}\right| \psi\right\rangle, \\
& \bar{p}_{i}=\left\langle\psi\left|p_{i}\right| \psi\right\rangle, \\
& S_{i j}=\left\langle\psi\left|x_{i} x_{j}\right| \psi\right\rangle, \\
& K_{i j}=\left\langle\psi\left|p_{i} p_{j}\right| \psi\right\rangle, \\
& Q_{i j}=\left\langle\psi\left|x_{i} p_{j}\right| \psi\right\rangle .
\end{aligned}
$$

The matrices $S$ and $K$ are real and symmetric. It is convenient to decompose $Q$ in the real symmetric part $Z$ ("vibrational momentum"), the real antisymmetric part $L$ ("angular momentum"), and the imaginary part

$$
Q=\frac{1}{2}(\boldsymbol{Z}+L+i I), \quad Q^{\dagger}=\frac{1}{2}(\boldsymbol{Z}-L-i I)
$$

with $Z_{i j}=\left\langle\psi\left|x_{i} p_{j}+p_{i} x_{j}\right| \psi\right\rangle, \quad L_{i j}=\left\langle\psi\left|x_{i} p_{j}-p_{i} x_{j}\right| \psi\right\rangle(1$ $-\delta_{i j}$ ), and $I$ the $3 \times 3$ unit matrix.

The dynamics of the center of mass and the mean momentum of the wave packet is well known. It follows Newton's equations

$$
\begin{aligned}
\dot{\bar{x}}_{i}=\bar{p}_{l} / m, \quad \dot{\bar{p}}_{i} & =-\left\langle\psi\left|\frac{\partial V(\mathbf{x})}{\partial x_{i}}\right| \psi\right\rangle \\
& =-\frac{\partial V(\overline{\mathbf{x}})}{\partial \bar{x}_{i}}+\cdots,
\end{aligned}
$$


where the ellipsis represents the corrections, which are due to the finite width.

In this article, we focus our attention on the dynamics of the "shape" of wave packets. Therefore we shall restrict ourselves to the second-order moments described by $S, K$, and $Z$, which represent the mean-square widths.

We shall first discuss the description of wave packets in terms of moments, in particular the representability problem and the uncertainty relations expressed by the second-order moments. Then we shall consider the equations of motion, especially those for the harmonicoscillator Hamiltonian. Finally, we shall establish initial conditions such that the wave packet propagates with conservation of shape.

\section{REPRESENTABILITY AND UNCERTAINTY RELATIONS}

The question arises whether for a given reduced density matrix $D$ there exists a wave function $|\psi\rangle$ or an ensemble such that the expectation values of corresponding operators yield $D$. This problem is known in the literature as the "representability problem." For a general set of operators $A_{a}$ the representability problem has not been solved in an explicit form. In our case the answer is simple because, as will be shown below, our density matrix can be transformed into the usual reduced one-body density matrix for which the representability conditions are well known.

Let us study the representability problem of a particular density matrix in its center-of-mass system such that $\bar{x}_{i}=0, \bar{p}_{i}=0$ since no generality is lost by this. Then we need only a smaller density matrix:

$$
D=\left[\begin{array}{cc}
S & Q \\
Q^{\dagger} & K
\end{array}\right]
$$

For formal discussions and proofs it is convenient to work in a harmonic-oscillator basis and express the operators $x_{i}$ and $p_{i}$ in terms of the familiar creation and annihilation operators for harmonic quanta $a_{i}^{\dagger}=\left(x_{i}-i p_{i}\right) / \sqrt{2}$ and $a_{i}=\left(x_{i}+i p_{i}\right) / \sqrt{2}$. In this representation, one can imagine, instead of one particle in full one-body Hilbert space, an indefinite number of bosons (harmonic quanta) with only three basic states available. The density matrix in the "many-boson" representation is

$$
\widetilde{D}=\left[\begin{array}{cc}
\widetilde{S} & \widetilde{Q} \\
\widetilde{Q}^{\dagger} & \widetilde{K}
\end{array}\right]=\left(\begin{array}{cc}
\left\langle\psi\left|a_{i}^{\dagger} a_{j}\right| \psi\right\rangle & \left\langle\psi\left|a_{i}^{\dagger} a_{j}^{\dagger}\right| \psi\right\rangle \\
\left\langle\psi\left|a_{i} a_{j}\right| \psi\right\rangle & \left\langle\psi\left|a_{i} a_{j}^{\dagger}\right| \psi\right\rangle
\end{array}\right)
$$

In the $(x, p)$ representation the reduction of the density matrix corresponds to the truncation of the complete one-body density matrix of the one-body system to a submatrix $D_{a b}$ defined by a small number of operators $A_{a}$. In the $\left(a, a^{\dagger}\right)$ representation this corresponds to the reduction of the many-boson density matrix to the reduced one-boson density matrix $\widetilde{S}$ and the "pairing correlation" matrix $\widetilde{Q}^{\dagger}$. Both reductions are equivalent. Since the transformation between $D$ and $\widetilde{D}$ is unitary, the transformed density matrix will have the same eigenvalue spectrum.
We subject to the representability test density matrices with correct symmetry ( $S$ and $K$ are real symmetric, $\operatorname{Im} Q=\frac{1}{2} i I$; or equivalently, $\widetilde{S}$ is Hermitian, $\widetilde{K}=\widetilde{S}+I$, and $\widetilde{Q}$ is symmetric). Then the necessary and sufficient condition for ensemble representability is [5] that the matrix $\widetilde{D}$ be non-negative. Equivalently the necessary and sufficient condition is that the matrix $D$ be non-negative, since the unitary transformation between them conserves the eigenvalue spectrum. We shall make extensive use of this condition.

Non-negative matrices form a convex set. It is an interesting question to see what are the exposed points of this convex set. It has been proved [6] that the exposed points are characterized by having the maximum possible number of zero eigenvalues. For the harmonic-oscillator ground state, the density matrix obviously has three zero eigenvalues, since the three annihilation operators eliminate such a state so that the upper three rows and the left three columns in the matrix $\widetilde{D}$ vanish. It is impossible to have more than three zero eigenvalues, since the creation and annihilation operators cannot simultaneously annihilate a state.

For any other Gaussian

$$
N \exp \left(-\sum_{i, j} x_{i} F_{i j} x_{j}\right)
$$

with

$$
F=\frac{1}{2}\left(U^{-1}-i V\right) .
$$

With $U$ symmetric positive definite and $V$ symmetric, we can define a canonical basis in which it represents the oscillator ground state leading to three zero eigenvalues of $D$. We conclude that all exposed points are just all possible Gaussians. The nonexposed surface points and the interior points of the convex set (where $D$ has less than three zero eigenvalues) correspond to all other wave functions and/or ensembles.

It is useful to evaluate the matrix $D$ for a general Gaussian parametrized as above. One gets

$$
\begin{aligned}
& S=\frac{1}{2} U, \quad K=\frac{1}{2}\left(V U V+U^{-1}\right), \\
& Z=\frac{1}{2}(U V+V U), \quad L=\frac{1}{2}(U V-V U) .
\end{aligned}
$$

We see that if $F$ is real the vibrational momentum $Z$ and the angular momentum $L$ are zero.

Since the matrix $D$ is non-negative, each of its submatrices (along the diagonal) is also non-negative, for example,

$$
\left[\begin{array}{cc}
S_{i i} & \frac{1}{2}\left(Z_{i i}+i\right) \\
\frac{1}{2}\left(Z_{i i}-i\right) & K_{i i}
\end{array}\right] \geq 0 .
$$

Then $S_{i i} K_{i i}-\frac{1}{4}\left(Z_{i i}^{2}+1\right) \geq 0$. We get immediately the uncertainty relation

$$
S_{i i} K_{i i} \geq \frac{1}{4}\left(Z_{i i}^{2}+1\right),
$$

or the more familiar (less restrictive) form by dropping the positive term $Z_{i i}^{2}$.

One can always make a canonical transformation from $x_{i}$ and $p_{i}$ to some new basis (for a fixed $i$ ) such that it di- 
agonalizes the real part of the above submatrix, giving $Z_{i i}=0$. If the equality sign is satisfied, one manifestly has the minimum uncertainty wave packet in this new canonical representation, while in the old $(x, p)$ representation it seemed to be nonminimal. It is worth noting that the uncertainty relation can be considered as a necessary (but not sufficient) representability condition for the matrix $D$.

More general than the uncertainty product for individual $i$ is the "uncertainty determinant," i.e., the determinant of the real part $\operatorname{Re} D$ of $D$. It is easy to see that this quantity also satisfies an uncertainty relation. Indeed, there exists a linear canonical transformation between $x_{i}$ and $p_{i}$ which diagonalizes $\operatorname{Re} D$. For the new density matrix $D^{\prime}$ then the uncertainty determinant appears as the product of three uncertainty products and so has to satisfy the inequality $\operatorname{Det}\left(\operatorname{Re} D^{\prime}\right) \geq\left(\frac{1}{4}\right)^{3}$. Since the linear canonical transformation is actually a symplectic transformation [7], it conserves the determinant and hence the inequality is satisfied also in the original basis. The equality applies for any Gaussian. The consequence is that any Gaussian wave packet can be considered as a minimum uncertainty wave packet if it is viewed in the appropriate canonical basis and if the "uncertainty determinant" is taken to be the measure of uncertainty.

\section{TIME EVOLUTION OF THE WAVE PACKET}

We shall study the time evolution of the density matrix using the equation of motion

$$
\dot{D}_{a b}=-i\left\langle\psi\left|\left[A_{a}^{\dagger} A_{b}, H\right]\right| \psi\right\rangle
$$

or, in case of an ensemble,

$$
\dot{D}_{a b}=-i \operatorname{Tr}\left(\rho\left[A_{a}^{\dagger} A_{b}, H\right]\right) .
$$

We shall consider Hamiltonians which are at most bilinear in $\mathbf{x}$ and $\mathbf{p}$ (free particle, free fall, or harmonic oscillator). We shall use dimensionless quantities as before (i.e., units are such that $\hbar=1$, the particle mass $m=1$, and the characteristic length $b$ and time $\omega^{-1}$ are also unity). The system of equations for the chosen density matrix is closed. The equations of motion for the second moments are closed since the corresponding operators (bilinear in $\mathbf{x}$ and p) form a closed algebra and the Hamiltonian consists only of these operators. This algebra is well known; it is the symplectic algebra $\mathrm{sp}(6 R)$ for three canonical degrees of freedom and is particularly useful for systems of harmonic oscillators.

One can study independently the motion of $\bar{x}, \bar{p}$ and independently the evolution of the widths $S, K, Q$ without the need for information about the higher moments of the shape of the wave packet.

For the free particle, with $H=\frac{1}{2} \mathbf{p}^{2}$, we get

$$
\dot{S}=Z, \quad \dot{K}=0, \quad \dot{Z}=2 K, \quad \dot{L}=0
$$

with the solution

$$
\begin{aligned}
& S=S_{0}+Z_{0} t+K_{0} t^{2}, \\
& K=K_{0},
\end{aligned}
$$

$$
\begin{aligned}
& Z=Z_{0}+2 K_{0} t, \\
& L=L_{0} .
\end{aligned}
$$

It is well known that the dispersion in momentum $K$ remains constant due to the momentum conservation while the dispersion in the coordinate $S$ spreads in time, so that the uncertainty product increases in time. We have remarked, however, in Sec. II that the uncertainty determinant of a Gaussian remains constant so that in an appropriate (time-dependent) canonical basis the uncertainty product also remains constant.

In the case of free fall, with $H=\frac{1}{2} \mathbf{p}^{2}+\mathbf{g} \cdot \mathbf{x}$, the center of mass of the wave packet falls classically while the width parameters $S, K, Q$ evolve exactly the same way as for a free particle, a consequence of Galilei invariance of the problem.

For the harmonic oscillator $H=\frac{1}{2} \mathbf{p}^{2}+\frac{1}{2} \mathbf{x}^{2}$, we get

$$
\dot{S}=Z, \quad \dot{K}=-Z, \quad \dot{Z}=-2 S+2 K, \quad \dot{L}=0
$$

with the solution

$$
\begin{aligned}
& S=\frac{1}{2}\left(S_{0}+K_{0}\right)+\frac{1}{2}\left(S_{0}-K_{0}\right) \cos 2 t+\frac{1}{2} Z_{0} \sin 2 t, \\
& K=\frac{1}{2}\left(S_{0}+K_{0}\right)-\frac{1}{2}\left(S_{0}-K_{0}\right) \cos 2 t-\frac{1}{2} Z_{0} \sin 2 t, \\
& Z=-\left(S_{0}-K_{0}\right) \sin 2 t+Z_{0} \cos 2 t, \\
& L=L_{0} .
\end{aligned}
$$

We see that all widths oscillate around a mean value at double oscillator frequency. The matrices $2 S, 2 K$, and $Z$ oscillate with the same amplitude (in our dimensionless units) but $Z$ is advanced in phase by $\frac{1}{2} \pi$ and $K$ by $\pi$ with respect to $S$. We can write $K(t)=S\left(t+\frac{1}{2} \pi\right)$, $Z(t)=S\left(t+\frac{1}{4} \pi\right)-K\left(t+\frac{1}{4} \pi\right)$. We also note that the angular momentum $L$ is conserved.

\section{RIGIDLY ROTATING WAVE PACKETS}

We are particularly interested in "rigid" wave packets which do not change shape with time. Since we restricted ourself to a partial information, the reduced density matrices containing only first- and second-order moments, we can describe only the time evolution of these moments. The first-order moments (the center of mass of the wave packet) of course move along the classical trajectory defined by Newton's law. We shall consider the shape inasmuch as it is characterized by the second-order moments (relative to the center of mass) and we shall ignore the fine time variation of the shape as seen by higher-order moments.

For the free particle one can see from the solution of (20) that $S, K, Z$, and $L$ are constant if $Z_{0}$ and $K_{0}$ are zero. This is, however, not possible (not representable) since it would violate the uncertainty relation. For the harmonic oscillator, on the other hand, constant rigid wave packets do exist when $S_{0}=K_{0}, Z_{0}=0$ as is clear from (25). This may surprise the reader who is familiar only with the rigid wave packet $S_{0}=K_{0}=\frac{1}{2} I, Z_{0}=0$ (Glauber state). We get here the whole family of solutions with $\left(S_{0}\right)_{i j}=\left(K_{0}\right)_{i j}=\lambda_{i} \delta_{i j}, \lambda_{i} \geq \frac{1}{2}$; it represents, for example, ensembles of two Gaussians: one with large $S_{0}$ 
and small $K_{0}$ and the other vice versa, so that both Gaussians oscillate in opposite phase and in the ensemble average the oscillation of $S$ and $K$ average out. This family is also representable by pure states. The $n$th excited stationary states (eigenstates) $|n\rangle$ of the harmonic oscillator having $S_{0}=K_{0}=\left(n+\frac{1}{2}\right) I$, superpositions thereof can have an arbitrary value of the quantity $S_{0}$. For superpositions $|\psi\rangle=\sum_{n} c_{n}|n\rangle$ which only contain components differing in $n$ by more than 2, there will be no cross terms in the second-order moments due to the selection rule of harmonic-oscillator eigenstates. Therefore the reduced density matrix will look the same as for an ensemble of stationary states with probabilities $\left|c_{n}\right|^{2}$ which is obviously stationary (constant). Of course, one would discover the difference between such a pure state and an ensemble if one looked at higher-order moments.

The next question is whether there also exist, in addition to these constant rigid wave packets, "rotating" rigid wave packets, i.e., for which the shape matrix $S$ will remain constant in some "body-fixed" frame. They are best described in a rotating frame where the harmonic oscillator is governed by the Hamiltonian

$H^{\prime}=H-\sum_{k, l} \Omega_{k l} x_{k} p_{l}=\frac{1}{2} \mathbf{p}^{2}+\frac{1}{2} \mathbf{x}^{2}-\operatorname{Tr}[\Omega(\mathbf{x} \times \mathbf{p})]$,

where $\Omega$ is the antisymmetric tensor of instantaneous angular velocity.

The equations of motion that one derives from $H^{\prime}$ are

$$
\begin{aligned}
& \dot{S}=Z-[S, \Omega], \\
& \dot{K}=-Z-[K, \Omega], \\
& \dot{Z}=-2 S+2 K-[Z, \Omega], \\
& \dot{L}=-[L, \Omega] .
\end{aligned}
$$

We define a rigidly rotating wave packet as one for which the spatial shape of the second-order moments of the probability distribution remains constant in time, i.e., $S=S_{0}$; so we require $\dot{S}=0$. Now, from the temporal relations between $S, K$, and $Z$ given in Sec. III, it follows immediately that $K$ and $Z$ will also be constant matrices. So the requirement of shape conservation leads to the following relations:

$$
\begin{aligned}
& Z=[S, \Omega]=-[K, \Omega], \\
& K=S+\frac{1}{2}[Z, \Omega]=S+\frac{1}{2}[[S, \Omega], \Omega], \\
& 0=[L, \Omega] .
\end{aligned}
$$

One can, by elimination, obtain the condition for $S$ in the following form:

$$
4[S, \Omega]+[[[S, \Omega], \Omega], \Omega]=0 .
$$

\section{A. Two-dimensional rotating wave packets}

It is easy to get the solution of (38) in two dimensions. In this case we can conveniently use the well-known Pauli matrices. We write $S=A I+B \sigma_{z}$ and $\Omega=\omega i \sigma_{y}$. Then $Z=[S, \Omega]=2 B \omega \sigma_{x}$ and further $[[[S, \Omega], \Omega], \Omega]$ $=-8 B \omega^{3} \sigma_{x}$. Now the equation (38) for $S$ has the simple form

$$
B \omega \sigma_{x}-B \omega^{3} \sigma_{x}=0 .
$$

This yields the solution for the angular velocity

$$
|\omega|=1
$$

which shows that the rotation is uniform. The two possible signs for $\omega$ distinguish both possible directions of rotation. One also gets

$$
K=S+\frac{1}{2}[Z, \Omega]=A I-B \sigma_{z}
$$

and

$$
L=l i \sigma_{y} .
$$

We have obtained a three-parameter family of rotating rigid wave packets with arbitrary parameters $A, B$, and $l$ which are only constrained by representability inequalities.

We would also like to find the subfamily of rigidly rotating Gaussians. According to the theorem on exposed points of our convex set of density matrices (see Sec. II), the $D$ matrix of Gaussians in two dimensions should have two zero eigenvalues. The corresponding two conditions reduce the three-parameter family to a one-parameter family of rigidly rotating Gaussians. The eigenvalues of the four-dimensional matrix $D$ can easily be determined. They are $2 A+l \pm \sqrt{16 B^{2}+1}$ and $2 A-l \pm 1$. They appear pairwise and because of the positiveness of $D$ only the smaller can be set equal to zero. So we get

$$
l=2 A-1, \quad B=\sqrt{A\left(A-\frac{1}{2}\right)} .
$$

The second condition follows after expressing the value of $l$ from the first. From these can also be easily deduced the elegant form

$$
S_{11}^{-1}+S_{22}^{-1}=\operatorname{Tr}\left(S^{-1}\right)=4 .
$$

It is easy to get the parameters of the Gaussian by inverting the Eqs. (15):

$$
U=2 S, \quad V=(B / A) \sigma_{x} .
$$

\section{B. Three-dimensional rotating wave packets}

In three dimensions the algebra is more involved. We can again suppose at time zero the matrix $S$ to be diagonal due to a proper choice of the coordinate axes. Then writing $\Omega_{i j}=e_{i j k} \Omega_{k}$ the rigidity condition (38) can easily be written out in components. For the diagonal elements we find

$$
\begin{aligned}
& \left(S_{3}-S_{2}\right) \Omega_{1} \Omega_{2} \Omega_{3}=0, \\
& \left(S_{1}-S_{3}\right) \Omega_{1} \Omega_{2} \Omega_{3}=0, \\
& \left(S_{2}-S_{1}\right) \Omega_{1} \Omega_{2} \Omega_{3}=0 .
\end{aligned}
$$

For the off-diagonal elements we find

$$
\begin{aligned}
\left(4 S_{2}-3 S_{3}-S_{1}\right) \Omega_{1}^{2} \Omega_{3}- & \left(4 S_{1}-3 S_{3}-S_{2}\right) \Omega_{2}^{2} \Omega_{3} \\
& =4\left(S_{1}-S_{2}\right)\left(\Omega_{3}^{2}-1\right) \Omega_{3},
\end{aligned}
$$




$$
\begin{gathered}
\left(4 S_{3}-3 S_{1}-S_{2}\right) \Omega_{2}^{2} \Omega_{1}-\left(4 S_{2}-3 S_{1}-S_{3}\right) \Omega_{3}^{2} \Omega_{1} \\
=4\left(S_{2}-S_{3}\right)\left(\Omega_{1}^{2}-1\right) \Omega_{1}, \\
\left(4 S_{1}-3 S_{2}-S_{3}\right) \Omega_{3}^{2} \Omega_{2}-\left(4 S_{3}-3 S_{2}-S_{1}\right) \Omega_{1}^{2} \Omega_{2} \\
=4\left(S_{3}-S_{1}\right)\left(\Omega_{2}^{2}-1\right) \Omega_{2} .
\end{gathered}
$$

The solutions of this set of equations can be discussed systematically by distinguishing the four possible cases in which all, two, one, or none in the $\Omega$ components are taken to be zero.

In the first case the above equations are trivial identities. In fact we are dealing with constant rigid wave packets discussed at the beginning of this section.

In the second case we suppose only $\Omega_{1}$ to be different from zero. Now again the diagonal equations are identically satisfied and the off-diagonal ones immediately lead to the requirement $\left|\Omega_{1}\right|=1$ if $S_{2} \neq S_{3}$. This case reduces to the two-dimensional one discussed earlier. One takes a rigidly rotating wave packet in two dimensions and a constant nonspreading one in the third dimension. Such a wave packet will rotate around one of the major axes of the quadrupole tensor.

For the third case, we assume both $\Omega_{2}$ and $\Omega_{3}$ to be nonzero. Again the diagonal equations are fulfilled. Now only one of the off-diagonal equations is an identity; the other two express the angular velocity components in terms of the $S$ elements:

$$
\begin{aligned}
& \Omega_{2}^{2}=4\left(S_{1}-S_{2}\right) /\left(S_{3}-S_{2}\right), \\
& \Omega_{3}^{2}=4\left(S_{3}-S_{1}\right) /\left(S_{3}-S_{2}\right) .
\end{aligned}
$$

We see from these relations that now $|\Omega|=2$. The other moments $Z$ and $K$ are related to $S$ by Eqs. (31) and (32). The angular momentum $L$ is directed along $\Omega$ but its magnitude is arbitrary. This case produces a genuinely three-dimensional four-parameter family of wave packets rotating uniformly about an axis in one of the major planes of the quadrupole tensor with angular velocity 2 .

Finally, the fourth case in which all $\Omega$ components are finite requires that $S_{1}=S_{2}=S_{3}$ and this reduces the offdiagonal equations to an identity such that the $\Omega$ vector is undetermined, consistent with the spherical shape of the wave packet. This case represents the trivial way in which in three dimensions one makes a rigid wave packet by combining three one-dimensional states of constant shape, one for each direction in space.

If we are interested in finding the Gaussian wave packets that conserve shape in three dimensions, we must further impose the condition that three of the eigenvalues of the six-dimensional density matrix vanish. In this way the above families are reduced to the trivial spherical case of three Glauber states and to two interesting families of Gaussian wave packets. The first family is essentially two dimensional, has one free parameter, and all its members rotate with angular velocity equal to the basic oscillator frequency; the second is a genuinely three-dimensional one-parameter family with an angular velocity twice the oscillator frequency. In terms of the parametrization by $U$ and $V$ given before, it can be established [4] that in the latter case the three moments must be related by the formulas

$$
\operatorname{Tr}\left(S^{-1}\right)=6, \quad S_{1}=2 S_{2} S_{3}
$$

\section{CONCLUSION}

We have investigated the dynamics of the second-order moments of the coordinate-momentum phase-space distribution of general wave packets under the action of harmonic forces. We have singled out those initial conditions that give rise to propagation with constant secondorder spatial moments and obtained two families of shape-conserving wave packets. They contain as special cases two families of Gaussian wave packets, which in turn contain the spherical Glauber states as singular elements.

The density-matrix approach used here is particularly useful because it provides a methodical scheme for classifying the relevant operators or, alternatively, the relevant wave-packet parameters, and at the same time it puts the analysis in the context of a well-established mathematical subject.

The density-matrix formalism is also convenient for discussing the anharmonic effects. For a harmonicoscillator Hamiltonian, the equations of motion for moments of a given order are decoupled from those of any other order; therefore one can get an exact solution in the framework of a chosen reduced density matrix alone. Anharmonic terms in the Hamiltonian couple moments of different order and the exact solution, even if it is a pure state, projected onto the reduced density matrix $D$ is no longer an exposed point and it looks like an ensemble density matrix. One can observe the anharmonic effects of the exact solution by watching how $D$ moves from an initially exposed point (Gaussian wave packet) into the interior. The measure of anharmonicity could be, e.g., the sum of the three smallest eigenvalues of $D$, which is zero for a Gaussian exposed point and positive for interior points. On the other hand, for approximate solutions of the anharmonic problem within $D$ alone, the use of ensembles rather than pure states alone emphasizes the imprecision of the approximation. If a solution is approximate, it can as well be further stochastically smeared out by taking a statistical ensemble rather than a pure state. This may smooth some unwanted fluctuations due to imprecisions of the approximation.

The ideas of shape dynamics and density matrices can also be extended to many-body systems. There are also phenomena such as collective motion that can be described by the time evolution of second-order moments, such as $S_{i j}^{N}=\operatorname{Tr}\left(\rho^{N} \sum_{v} x_{i}^{v} x_{j}^{v}\right)$, where $\rho^{N}$ is the $N$-body density operator. Because they involve one-body operators only, they can be expressed in terms of the reduced onebody density operator

$$
\begin{aligned}
& \rho^{1}\left(\mathbf{x}, \mathbf{x}^{\prime}\right) \\
& \quad=\int \rho^{N}\left(\mathbf{x}, \mathbf{x}^{2}, \ldots, \mathbf{x}^{N} ; \mathbf{x}^{\prime}, \mathbf{x}^{2}, \ldots, \mathbf{x}^{N}\right) d \mathbf{x}^{2}, \ldots, d \mathbf{x}^{N}
\end{aligned}
$$

in the form $S_{i j}^{N}=\operatorname{Tr}\left(\rho^{1} x_{i} x_{j}\right)$. Although there are some 
differences between the one-body density matrix and the $N$-body density matrix related to the representability of $\rho^{1}$, the foregoing analysis remains valid. Of course, even if $\rho^{1}$ corresponded to a pure state, it would in general look like an ensemble density operator. The matrix of second-order moments can be considered as doubly reduced, first with respect to the reduction from $N$-body to one-body and second with respect to the truncation from the full one-body reduced density matrix to a partial in- formation contained in the expectation values of the chosen operators, bilinear in $x$ and $p$.

\section{ACKNOWLEDGMENTS}

P. Van Leuven wishes to thank the Institute J. Stefan and the University of Ljubljana for their hospitality. Interesting discussions with F. Arickx and J. Broeckhove are gratefully acknowledged.
[1] E. Schrödinger, Naturwissenschaften 14, 664 (1926).

[2] R. J. Glauber, Phys. Rev. 130, 2529 (1963).

[3] A. Perelomov, Generalized Coherent State and Their Applications (Springer-Verlag, Berlin, 1986).

[4] F. Arickx et al., Phys. Rev. A 43, 1211 (1991).

[5] A. J. Coleman, in Reduced Density Matrices with Applications to Physical and Chemical Systems, edited by A. J. Coleman and R. M. Erdahl, Queen's Papers on Pure and Applied Mathematics Vol. 11 (Queen's University,
Kingston, Ontario, Canada, 1968), p. 2.

[6] M. Rosina, in Reduced Density Operators with Applications to Physical and Chemical Systems II, edited by R. M. Erdahl, Queen's Papers on Pure and Applied Mathematics Vol. 40 (Queen's University, Kingston, Ontario, Canada, 1974), p. 57.

[7] H. Goldstein, Classical Mechanics, 2nd ed. (AddisonWesley, Reading, MA, 1980), Sec. 9-3. 